\title{
SECONDARY BIOACTIVE METABOLITE GENE CLUSTERS IDENTIFICATION OF ANTICANDIDA-PRODUCING Streptomyces Sp. GMR22 ISOLATED FROM WANAGAMA FOREST AS REVEALED BY GENOME MINING APPROACH
}

\author{
Camelia Herdini ${ }^{1,5_{*}}$, Sofia Mubarika ${ }^{1}$, Bambang Hariwiyanto ${ }^{1,5}$, Nastiti Wijayanti $^{1}$, \\ Akira Hosoyama ${ }^{2}$, Atsushi Yamazoe ${ }^{2}$, Hideaki Nojiri ${ }^{3}$, Jaka Widada ${ }^{4 *}$
}

${ }^{1}$ Graduate School of

Biotechnology, Universitas Gadjah Mada, Barek Utara, Yogyakarta, Indonesia 2Biological Resource Center, Nat Inst of Technology and Evaluation, Nishihara, Shibuya-ku, Tokyo, Japan 3Biotechnology Research Center, The University of Tokyo, Bunkyo-ku, Tokyo, Japan

${ }^{4}$ Dept of Agricultural Microbiology, Universitas Gadjah Mada, Bulaksumur, Yogyakarta, Indonesia 5Otorhinolaryngology Head and Neck Surgery, Faculty of Medicine, Universitas Gadjah Mada, Sekip, Yogyakarta, Indonesia

Submitted: $09-08-2016$

Revised: 08-10-2016

Accepted: $15-12-2016$

*Corresponding author Jaka Widada

Email:

jwidada@ugm.ac.id

\begin{abstract}
Streptomyces are a group of Gram-positive bacteria belonging to the Actinobacteria class, which are among the most important bacteria for producing secondary bioactive metabolites such as antibiotics, chemotherapeutics, insecticides and other high-value chemicals. Genome mining of gene clusters that encode the biosynthetic pathways for these metabolites has become a key methodology for novel compound discovery. Recently, we have isolated the Streptomyces sp. GMR22 from Cajuput rhizospheric soil at Wanagama Forest, Indonesia. GMR22 produced a secondary metabolite that inhibited Candida albicans with IC $_{50}$ of $62.5 \mu \mathrm{g} / \mathrm{mL}$. The objective of this work was to reveal the novel secondary metabolites from GMR22 by genome mining approach. The antiSMASH 3.0 was used to predict gene clusters that encode the biosynthetic pathways of secondary metabolites in the genome of GMR22, and their core chemical structures. The phylogenomic analysis showed that GMR22 was closely related to Streptomyces bingchenggensis BCW1, as well as to the large genome size (9.5-12.7Mbp) groups of Streptomyces. AntiSMASH 3.0 analysis revealed that the genome of Streptomyces sp. GMR22 harbored at least 63 gene clusters that encode the biosynthetic pathways of secondary metabolites. It was the highest number of gene clusters had been observed among the members of Streptomyces groups, with polyketide synthetase (PKS) was predicted as the major groups of the identified gene cluster products. The results suggested that GMR22 could be a strong potential candidate for secondary bioactive metabolites source.
\end{abstract}

Key words: Streptomyces sp. GMR22, antifungal, genome sequence, antiSMASH

\section{INTRODUCTION}

Streptomyces is a representative of a filamentous bacteria responsible for producing variety of valuable secondary metabolites, such as antibiotics, parasiticides, herbicides, and pharmacologically active substances, including antitumor agents and immunosuppressants (Waksman and Woodruff, 1940; Ditsler et al., 1992; Euverink, 1995; Newman et al., 2000; El Hassan et al., 2001; Bentley et al., 2002; Bibb, 2005; Quintana et al., 2008; Li et al., 2009; Doroghazi et al., 2014). Genome sequencing analyses of Streptomyces avermitilis (Omura et al., 2001), Streptomyces coelicolor A3(2) (Bentley et al., 2002), Streptomyces griseus IFO 13350 (Ohnishi et al., 2008), and Streptomyces bingchenggensis (Wang et al., 2010) revealed that Streptomyces possess an unexpected abundance of natural product biosynthetic gene clusters and thus that they have the potential to make many more compounds than previously thought.

Previously, we isolated several Streptomyces from rhizospheric soil at Wanagama Forest Indonesia (Nurjasmi et al., 2009). Streptomyces sp. GMR22 isolated from Cajuput rhizospheric soil produced strong antifungal secondary metabolite and inhibited Candida albicans with $\mathrm{IC}_{50}$ of $62.5 \mu \mathrm{g} / \mathrm{mL}$ (Nurjasmi et al., 2009; Alimuddin et al., 2010).

Recently, genomic discovery from some Streptomyces can be used as guidance to obtain new bioactive compounds. In addition, 
biosynthesis gene clusters mining their genomes becomes a key method to accelerate identification and characterization. The antibiotic and secondary metabolite analysis of the shell (antiSMASH) is a web server and stand-alone software, which combines automatic identification of secondary metabolite gene clusters in the genome sequence analysis of a large collection of algorithms-specific compounds (Medema et al., 2011; Weber et al., 2015). For the last two years, antiSMASH became a standard tool for analyzing the genomes of bacteria and fungi for their potential of secondary metabolites production (Blin et al., 2013). In this paper, we reported the results of the genome mining by using AntiSMASH for analyzing genome sequence of GMR22 and compared to the most closed related Streptomyces based on the analysis of whole genome phylogenetic (phylogenomic).

\section{MATERIAL AND METHODS}

Analysis of whole genome phylogenetic tree of GMR22

Streptomyces sp. GMR22 genome sequence analysis was done by Next Generation Sequencing (NGS) platforms using 454 pyrosequencing technology (454 GS FLX) and HiSeq1000 (Illumina). Whole-genome phylogenetic analysis (phylogenomic) was done by Neighbour-joining algorithm showed genome relationship of Streptomyces sp. GMR22 and 20 complete genome sequences of Streptomyces. Phylogeny tree was created using CV tree with a $k$-value of 6 (Xu and Hao, 2009) with Bacillus subtilis natto BEST195 as an outgroup and visualized with MEGA 6.06 (Tamura et al., 2013).

\section{Analysis of Genome Mining of Streptomyces Sp. GMR22}

Identification, annotation, and analysis of gene cluster involved in the biosynthesis of secondary metabolites and predictions core structure produced done by using antiSMASH 3.0. (Medema et al., 2011; Weber et al., 2015). Available at HTTP://antism ash. secondarymetabolites.org. Prodigal and run secondary metabolite detection on all possible ORFs were chosen in the Gene finding options parameters.

\section{RESULTS AND DISCUSSION Phylogenomic of GMR22}

Genome sequence analysis results showed that the size of the genome of GMR22 was 11,42 Mbp. Based on the phylogenomic analysis showed that GMR22 isolate closely related to Streptomyces bingchenggensis BCW1 and clustered with strains S. violaceusniger Tu 4113, S. rapamycinicus NRRL 5491, and $S$. davawensis JCM 4913 that have large genome size (9.5-12.7Mbp).

\section{Secondary metabolite biosynthesis gene clusters in Streptomyces sp. GMR22} Secondary metabolism of microbes is a rich source of antibiotics, chemotherapy, insecticides and other high value chemicals. Genome mining group biosynthesis pathway genes coding for these metabolites has been a key methodology for the discovery of new compounds. To determine the gene cluster and bioactive secondary metabolites produced by strain GMR22 were then analyzed with AntiSMASH 3.0 program (Table I).

The results indicated that GMR22 harbored at least 63 gene clusters encoding the biosynthetic pathways of secondary metabolites. It was the highest number of gene clusters had been observed among the members of Streptomyces groups. It was also interesting that more than a third of the genes clusters are polyketide syntethase: 21 gene clusters contained of type I of pure polyketide syntethase (T1pks), 3 gene clusters contained of type II of polyketide syntethase (T2pks), 3 gene clusters contained of hybrid type III of polyketide syntethase, and 1 gene cluster of hybrid type I of polyketide syntethase.

Compared to the closest strain in phylogenomic tree (Figure 1), namely Streptomyces bingchenggensis BCW-1 (Wang et al., 2010), some secondary metabolites have some similarities. AntiSMASH analysis revealed that Streptomyces sp. GMR22 harboring at least 63 bioactive secondary metabolites gene clusters (12 NRPS and 28 PKS), while BCW-1 only has 53 bioactive secondary metabolites gene clusters (16 NRPS and 20 PKS). At least there are 9 of bioactive secondary metabolites produced by both strain, they were ectoin, hopene, actinomycin, skyllamycin, geldanamycin, desferrioxamine B, echosides, meridamycin and spores pigments. 


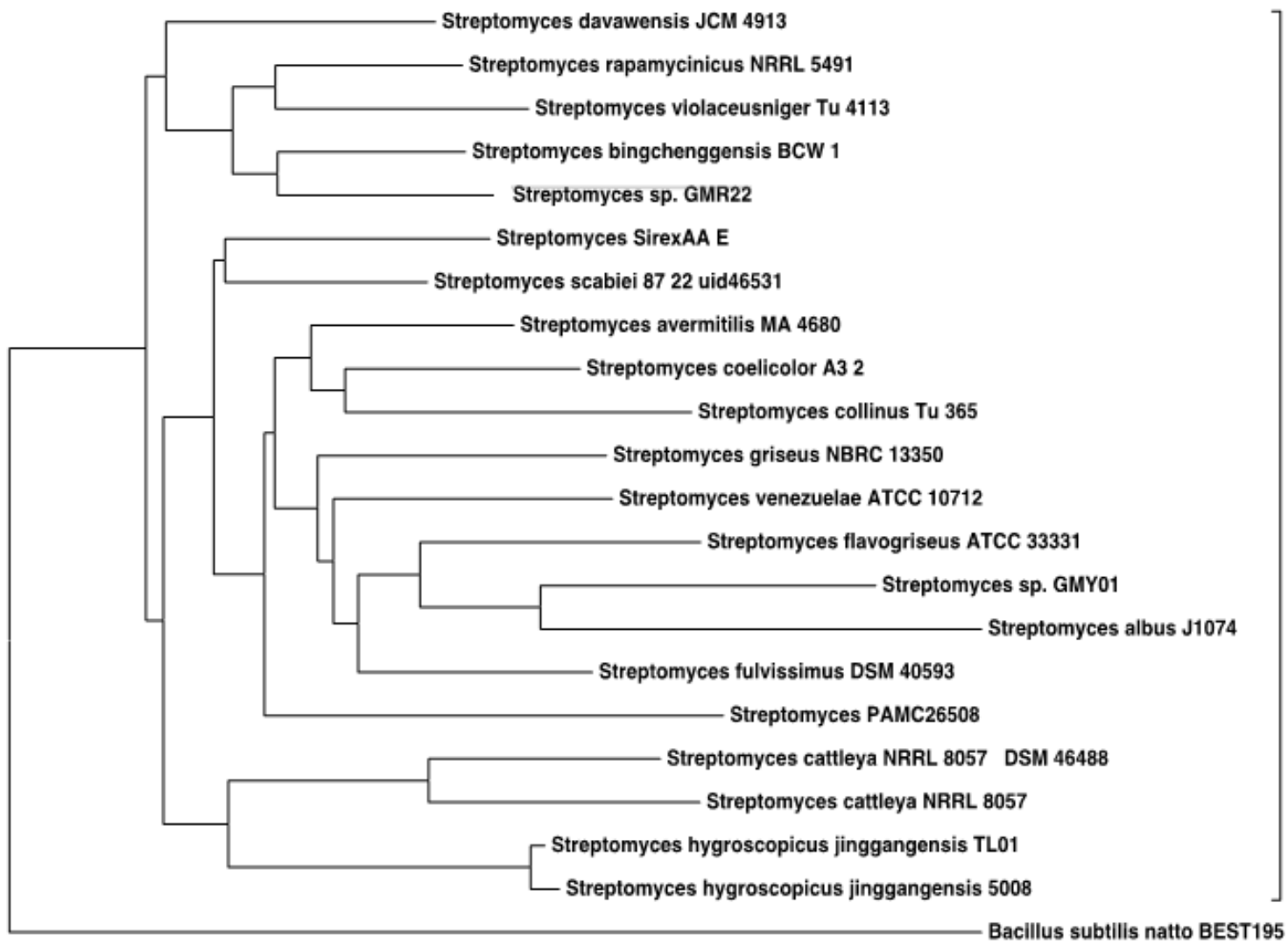

Figure 1. Analysis of whole-genome phylogenetic tree with Neighbour-joining algorithm showed the relationship of Streptomyces sp. GMR22 and other 20 completed genomic sequences of Streptomyces. Phylogeny tree was created using CVtree with a $k$-value of 6 (Xu and Hao, 2009) with Bacillus subtilis natto BEST195 as an out-group and was visualized with MEGA 6.06 (Tamura et al., 2013).

Table 1. Cluster of genes and bioactive secondary metabolites of Streptomyces sp. GMR22 based on the analysis of genome sequences with AntiSMASH 3.0 program cluster 1-14.

\begin{tabular}{lllll}
\hline Cluster & Type & Size (bp) & Most silmilar known cluster (\%) & MIB* \\
\hline Cluster 1 & T1pks & 40541 & Ansamitosin (21) & BGC0000020_c1 \\
Cluster 2 & Nrps & 53307 & Actinomycin (7) & BGC0000296_c1 \\
Cluster 3 & T1pks & 1553 & - & \\
Cluster 4 & T1pks & 26277 & Hygrocin (74) & BGC0000075_c1 \\
Cluster 5 & Lantipeptide & 23294 & Reveromycin (9) & BGC0000135_c1 \\
Cluster 6 & T1pks-Other & 123792 & Bafilomycin (50) & BGC0000957_c1 \\
& ks & & & \\
Cluster 7 & Bacteriocin- & 28221 & - & \\
& Lantipeptide & & & BGC0000957_c1 \\
Cluster 8 & T1pks & 47645 & Ansatrienin (7) & BGC0000242_c1 \\
Cluster 9 & T2pks & 42504 & Lysolipin (23) & \\
Cluster 10 & Terpene & 21101 & - & BGC0000374_c1 \\
Cluster 11 & Nrps- & 111619 & Hormaomycin (8) & \\
& Lantipeptide & & & - \\
Cluster 12 & T1pks & 45292 & - & - \\
Cluster 13 & Lassopeptide & 22386 & - & \\
Cluster 14 & Siderophore & 13368 & - &
\end{tabular}


Table II. Cluster of genes and bioactive secondary metabolites of Streptomyces sp. GMR22 based on the analysis of genome sequences with AntiSMASH 3.0 program cluster 15-55

\begin{tabular}{|c|c|c|c|c|}
\hline Cluster & Type & Size (bp) & Most silmilar known cluster (\%) & MIB* \\
\hline Cluster 15 & Butyrolactone & 10990 & - & \\
\hline Cluster 16 & Nrps & 88213 & Laspartomycin (53) & BGC0000379_c1 \\
\hline Cluster 17 & T1pks & 7156 & Chalcomycin (17) & BGC0000035_c1 \\
\hline Cluster 18 & T1pks & 12100 & ECO-02301 (32) & BGC0000052_c1 \\
\hline Cluster 19 & Terpene & 18680 & Hopene (53) & BGC0000663_c1 \\
\hline Cluster 20 & Ectoine & 10404 & Ectoine (75) & BGC0000853_c1 \\
\hline Cluster 21 & T1pks & 9684 & - & \\
\hline Cluster 22 & T1pks & 79794 & Elaiophylin (87) & BGC0000053_c1 \\
\hline Cluster 23 & Hserlactone & 20756 & Daptomycin (3) & BGC0000336_c1 \\
\hline Cluster 24 & Butyrolactone & 10936 & Meilingmycin (2) & BGC0000093_c1 \\
\hline Cluster 25 & Nrps-T3pks & 100064 & A47934 (44) & BGC0000290_c1 \\
\hline Cluster 26 & Nrps & 39174 & - & \\
\hline Cluster 27 & Nrps & 2918 & - & \\
\hline Cluster 28 & T1pks & 33977 & Geldanamycin (69) & BGC0000066_c1 \\
\hline Cluster 29 & T2pks & 42492 & Medermycin (50) & BGC0000245_c1 \\
\hline Cluster 30 & T1pks & 63580 & ECO-02301 (53) & BGC0000052_c1 \\
\hline Cluster 31 & Lassopeptide & 22646 & Polyxypeptin (8) & BGC0001036_c1 \\
\hline Cluster 32 & Terpene & 21074 & Merochlorin (4) & BGC0001083_c1 \\
\hline Cluster 33 & Siderophore & 13771 & - & \\
\hline Cluster 34 & Terpene & 25477 & Salinilactam (8) & BGC0000109_c1 \\
\hline Cluster 35 & T1pks & 114377 & Nemadectin (50) & BGC0000109_c1 \\
\hline Cluster 36 & $\begin{array}{l}\text { Arylpolyne- } \\
\text { Ladderane }\end{array}$ & 42384 & Skyllamycin (18) & BGC0000429_c1 \\
\hline Cluster 37 & Nrps & 44011 & Ochronotic (75) & BGC0000918_c1 \\
\hline Cluster 38 & Siderophore & 12058 & - & \\
\hline Cluster 39 & Bacteriocin & 11341 & - & \\
\hline Cluster 40 & T1pks & 62548 & Medermycin (13) & BGC0000245_c1 \\
\hline Cluster 41 & Siderophore & 11788 & DesferrioxamineB (100) & BGC0000941_c1 \\
\hline Cluster 42 & T3pks-Nrps & 57880 & Pheganomycin (38) & BGC0001148_c1 \\
\hline Cluster 43 & Other & 25323 & Echosides (52) & BGC0000340_c1 \\
\hline Cluster 44 & $\begin{array}{c}\text { Laddarane- } \\
\text { Arylpolyene- } \\
\text { Nrps }\end{array}$ & 104183 & Skyllamycin (46) & BGC0000429_c1 \\
\hline Cluster 45 & Nrps & 34119 & Meridamycin (18) & BGC0001011_c1 \\
\hline Cluster 46 & Nrps & 78699 & Leinamycin (4) & BGC0001101_c1 \\
\hline Cluster 47 & Terpene & 22238 & Brasilicardin (36) & BGC0000632_c1 \\
\hline Cluster 48 & Terpene & 20909 & - & \\
\hline Cluster 49 & Other & 42739 & Echosides (11) & BGC0000340_c1 \\
\hline Cluster 50 & Lantipeptide & 24154 & - & \\
\hline Cluster 51 & T1pks & 44652 & Ambruticin (14) & BGC0000014_c1 \\
\hline Cluster 52 & T1pks & 63597 & Elaiophylin (45) & BGC0000053_c1 \\
\hline Cluster 53 & T1pks & 77675 & Nigericin (94) & BGC0000114_c1 \\
\hline Cluster 54 & T1pks & 126001 & Indanomycin (21) & BGC0000079_c1 \\
\hline Cluster 55 & $\begin{array}{l}\text { Terpene- } \\
\text { T3pks-Nrps }\end{array}$ & 34999 & Lobophorin (20) & BGC0001183_c1 \\
\hline
\end{tabular}


Table 3. Cluster of genes and bioactive secondary metabolites of Streptomyces sp. GMR22 based on the analysis of genome sequences with AntiSMASH 3.0 program cluster 56-63.

\begin{tabular}{rllll} 
Cluster & \multicolumn{1}{c}{ Type } & \multicolumn{1}{c}{ Size (bp) } & $\begin{array}{l}\text { Most silmilar known cluster (\%) } \\
\text { Spore-pigment (75) }\end{array}$ & \multicolumn{1}{c}{ MIB* } \\
Cluster 56 & T2pks & 42516 & BGC0000271_c1 \\
Cluster 57 & Terpene & 21191 & 2-methylisoborneol (100) & BGC0000658_c1 \\
Cluster 58 & Otherks & 41029 & Galbonolides (20) & BGC0000065_c1 \\
Cluster 59 & Other & 44029 & - & \\
Cluster 60 & T1pks & 71853 & Meridamycin (55) & BGC0001011_c1 \\
Cluster 61 & T1pks & 45286 & Hygrocin (38) & BGC0000075_c1 \\
Cluster 62 & T1pks & 62304 & Oligomycin (38) & BGC0000117_c1 \\
Cluster 63 & T1pks & 46363 & Salinomycin (8) & BGC0000144_ct \\
\hline
\end{tabular}

*The Minimum Information on Biosynthetic Geneclusters (Genecluster repository)

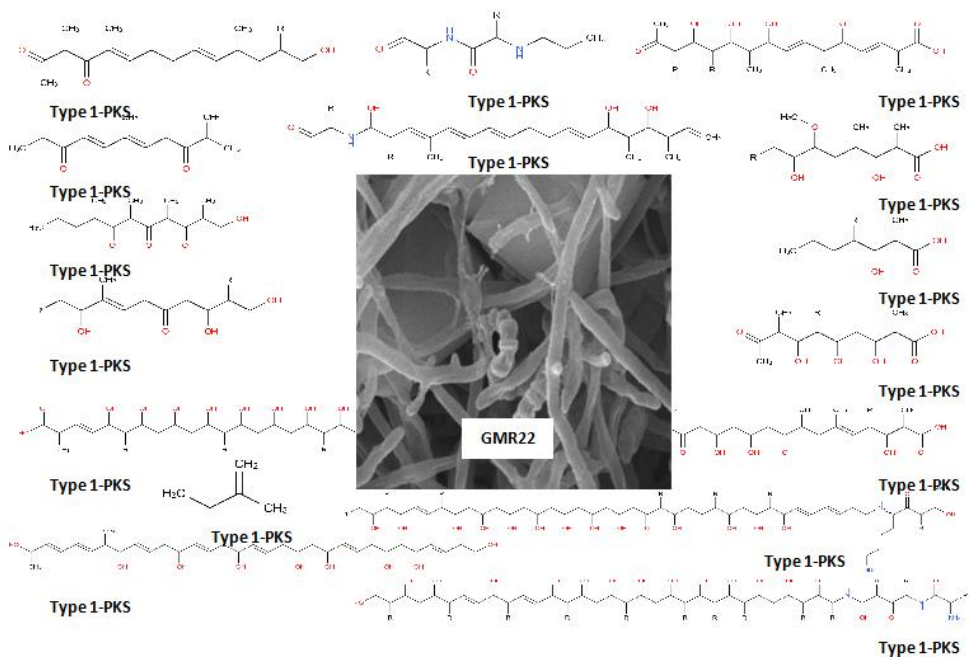

Figure 2. The chemical structure of the type 1-PKS predictable core group of secondary metabolites Streptomyces sp. GMR22 based on analysis of genome mining

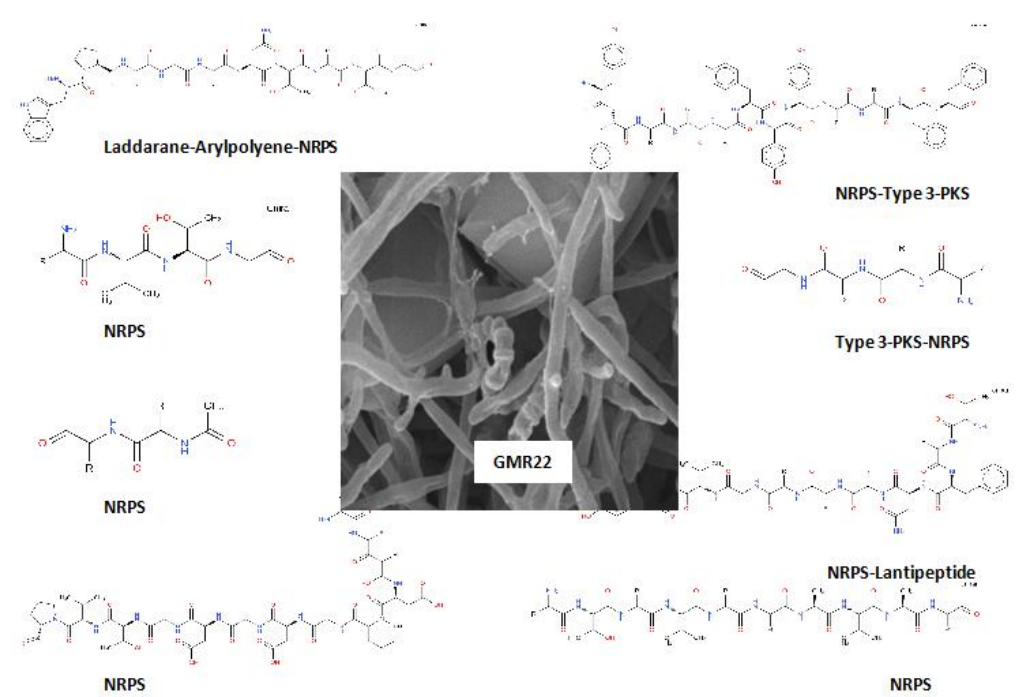

Figure 3. The chemical structure of the predictable core of NRPS group and NRPS hybrid of secondary metabolites Streptomyces sp. GMR22 based on analysis of genome mining 


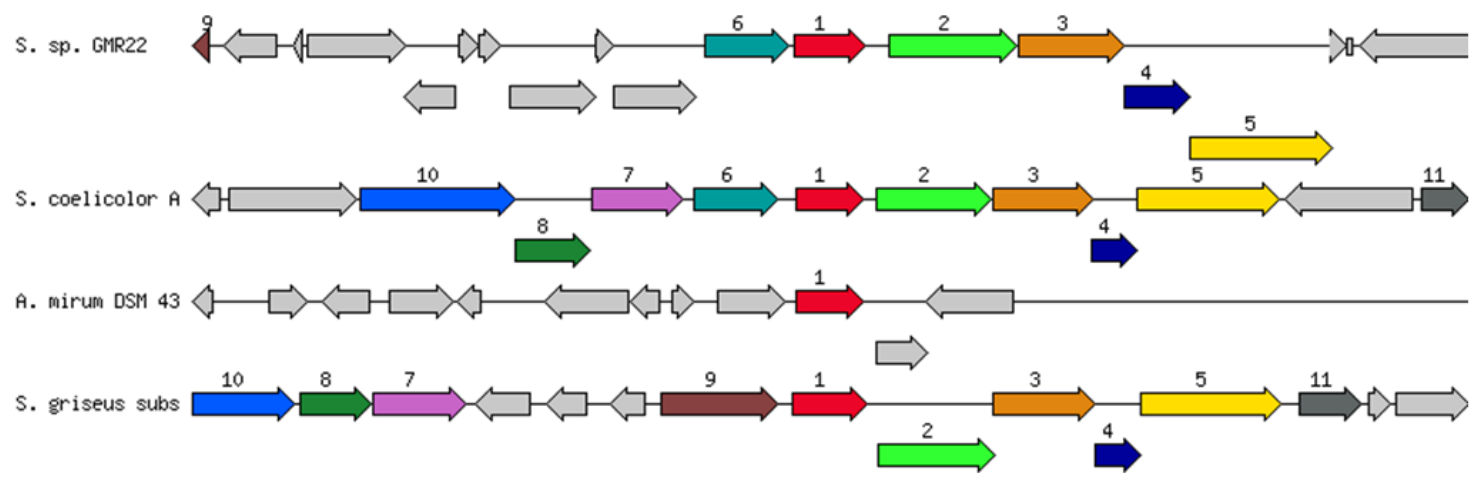

Figure 4. The cluster of genes Streptomyces sp. GMR22 involved in Desferrioxamine B production. S. sp GMR22: Streptomyces sp. GMR22; S. coelicolor: Streptomyces coelicolorA3(2); A. mirum DSM 43: Actinosynnema mirum DSM 43827; S. griseus: Streptomyces griseus subs sp. The number listed in the gene are as follows: 1. Hypothetical protein associated with desferrioxamine E biosynthesis; 2. Siderophore biosynthesis L-2,4-diaminobutyrate decarboxylase (DesA); 3. Siderophore biosynthesis protein, monooxygenase (DesB); 4. Siderophoresynthetase small component, acetyltransferase (DesC); 5. Siderophoresynthetase component, ligase (DesD); 6. Putative Desferrioxamine E transporter.

Here are some predictable chemical structures of secondary metabolites Streptomyces sp. GMR22 (Figures 2 and 3). The analysis was based on the genetic organization of secondary metabolites.

From figure 2, it can be seen that mostly the secondary metabolites of GMR22 dominated by type I-PKS. Polyketide is a large family of natural products found in bacteria, fungi, and plants. In addition, many clinically important drugs are included in this class, such as tetracycline, daunorubicin, erythromycin, rapamycin, and lovastatin. These compounds were biosynthesized from the precursor acylCoA by polyketide synthetase (PKS). Recent literature on polyketide biosynthesis suggests that polyketide synthases have much greater diversity in both mechanism and structure than the current type I, II and III paradigms. These examples serve as an inspiration for searching novel polyketide synthases to give new insights into polyketide biosynthesis and to provide new opportunities for combinatorial biosynthesis (Shen, 2003).

Secondary metabolites were also found in GMR22 with NRPS structure and hybrid NRPS with PKSs or others (Figure 3). However, NRPS structure is less compared to the class of PKSs compounds. Two secondary metabolites that encoded by type III of PKS were found in GMR22 as well. Type III of PKS is commonly found in fungi and plants. In general, type III of PKSs are involved in the biosynthesis of several lipid compounds and variety of secondary metabolite has some interesting characteristics, which does not belong to type III of PKS plants. In addition, many compounds produced by type III of PKS bacteria have significant biological functions in the interests of the pharmaceutical (Katsuyama and Ohnishi, 2012).

\section{Secondary metabolite biosynthesis gene clusters involved in anticancer production}

Based on the genome mining with antiSMASH 3.0, GMR22 has gene cluster for Desferrioxamine B which is $100 \%$ homologous with the most similar known cluster. Figure 4 shows a comparison of the gene cluster Desferrioxamine B GMR22 with Desferrioxamine B gene cluster of Streptomyces coelicolorA3(2) and Streptomyces griseus sub sp. griseus.

Desferrioxamine B

(MIB BGC0000941_c1) is a highly conserved gene cluster in Streptomyces genera (Figure 4). From this Figure, it can be seen that both genes that encode enzymes, as well as location and sequence of genes for GMR22, were similar to the gene cluster in Streptomyces coelicolor A3(2). The gene cluster comprises a set of 4 main enzymes (2 to 5) with essential functions for the siderophore biosynthesis: the gene 
encoding siderophore biosynthesis of L-2,4diaminobutyrate decarboxylase DesA (2), siderophore biosynthesis protein, monooxygenase DesB (3), siderophoresynthetase small component, and acetyltransferase DesC (4), and siderophoresynthetase component ligase DesD (5). There are 2 addition enzymes that may be also involved, namely: a protein associated with Desferrioxamine E biosynthesis (1) and Desferrioxamine E transporter (6). In GMR22 mostly the size of Desferrioxamine genes are longer than Desferrioxamine genes in Streptomyces coelicolor A3(2). Functional analysis comparison between of gene cluster from GMR22 and strain A3(2) would be an interesting study to prove whether the activities are the same. Desferrioxamine B is an iron chelator, which has a variety of clinical applications for patients with iron overload in terms of improving the quality of life and overall survival. In addition, Desferrioxamin B in the experiment showed potential anticancer in the cells of colon cancer (Salis et al., 2014).

\section{CONCLUSION}

Based on the analysis of phylogeny relationship on the whole genome sequence showed that GMR22 was closed related with Streptomyces bingchenggensis BCW1 and clustered with strains of Streptomyces which have large genome size (9.5-12.7Mbp). AntiSMASH analysis revealed that Streptomyces sp. GMR22 harbored at least 63 gene clusters that encode the biosynthetic pathways of secondary metabolites. It was the highest number of gene clusters had been observed among the members of Streptomyces groups, with PKS was predicted as the major groups of the identified gene cluster products. Further studies on Streptomyces sp. GMR22 will provide more insights into natural product biosynthesis potential of related Streptomyces.

\section{REFERENCES}

Alimuddin AW., Widada J., Mustofa NR., 2010. An actinomycete producing anticandida isolated from cajuput rhizosphere: partial identification of isolates and amplification of PKS-I genes. Indonesian J Biotechnol, 15, pp.1-8.
Bentley SD., Chater KF., Cerdeno-Tarraga, AM., Challis GL., Thomson NR., et al., 2002. Complete genome sequence of the model actinomycete Streptomyces coelicolor A3 (2). Nature, 417(6885), pp.141-147.

Bibb MJ., 2005. Regulation of secondary metabolism in streptomycetes. Current Opinion in Microbiology, 8, pp.208-215.

Blin K., Medema MH., Kazempour D., Fischbach MA., Breitling R., et al., 2013. antiSMASH 2.0-a versatile platform for genome mining of secondary metabolite producers. Nucleic acids research, p.gkt449.

Distler J., Mansouri K., Mayer G., Stockmann M., Piepersberg W., 1992. Streptomycin biosynthesis and its regulation in Streptomycetes. Gene, 115(1), pp.105111.

Doroghazi JR., Albright JC., Goering AW., Ju KS., Haines RR., Tchalukov KA., et al., 2014. A roadmap for natural product discovery based on large-scale genomics and metabolomics. Nature chemical biology, 10(11), pp.963-968.

El Hassan AM., Fahal AH., Ahmed AO., Ismail A., Veress, B., 2001. The immunopathology of actinomycetoma lesions caused by Streptomyces somaliensis. Transactions of the Royal Society of Tropical Medicine and Hygiene, 95(1), pp.89-92.

Euverink GJW., 1995. Aromatic amino acid biosynthesis in actinomycetes. Groningen: University of Groningen.

Katsuyama Y., Ohnishi Y., 2012. Type III polyketide synthases in microorganisms. Methods Ensymol, 515, pp.359-377.

Li MH., Ung PM., Zajkowski J., GarneauTsodikova S. Sherman DH., 2009. Automated genome mining for natural products. BMC bioinformatics, 10(1), p.1.

Medema MH., Blin K., Cimermancic P., de Jager V., Zakrzewski P., Fischbach MA., et al., 2011. antiSMASH: rapid identification, annotation, and analysis of secondary metabolite biosynthesis gene clusters in bacterial and fungal genome sequences. Nucleic acids research, 39(suppl 2), pp.W339-W346. 
Newman DJ., Cragg GM., and Snader KM., 2000. The influence of natural products upon drug discovery. Natural product reports, $17(3)$, pp.215-234.

Nurjasmi R., Widada J., Ngadiman N., 2009. Diversity of Actinomycetes at Several Forest Types in Wanagama I Yogyakarta and Their Potency as a Producer of Antifungal Compound. Indonesian Journal of Biotechnology, 14(2).

Ohnishi Y., Ishikawa J., Hara H., Suzuki H., Ikenoya M., et al., 2008. Genome sequence of the streptomycin-producing microorganism Streptomyces griseus IFO 13350. Journal of bacteriology, 190(11), pp.4050-4060.

Ōmura S., Ikeda H., Ishikawa J., Hanamoto A., Takahashi C., et al., 2001. Genome sequence of an industrial microorganism Streptomyces avermitilis: deducing the ability of producing secondary metabolites. Proceedings of the National Academy of Sciences, 98(21), pp.1221512220.

Quintana ET., Wierzbicka K., Mackiewicz P., Osman A., Fahal, AH., et al., 2008. Streptomyces sudanensis sp. nov., a new pathogen isolated from patients with actinomycetoma. Antonie Van Leeuwenhoek, 93(3), pp.305-313.

Salis O., Bedir A., Kilinc V., Alacam H., Gulten S., Okuyucu A., 2014. The anticancer effects of desferrioxamine on human breast adenocarcinoma and hepatocellular carcinoma cells. Cancer Biomarkers, 14(6), pp.419-426.

Shen B., 2003. Polyketide biosynthesis beyond the type I, II and III polyketide synthase paradigms. Current opinion in chemical biology, 7(2), pp.285-295.

Tamura K., Stecher G., Peterson D., Filipski, A., Kumar S., 2013. MEGA6: molecular evolutionary genetics analysis version 6.0. Molecular biology and evolution, 30(12), pp.2725-2729.

Waksman SA., Woodruff HB., 1940. Bacteriostatic and Bactericidal Substances Produced by a Soil Actinomyces. Experimental Biology and Medicine, 45(2), pp.609-614.

Weber T., Blin K., Duddela S., Krug D., Kim HU., et al., 2015. antiSMASH 3.0-a comprehensive resource for the genome mining of biosynthetic gene clusters. Nucleic acids research, 43(W1), pp.W237W243.

Wang XJ., Yan YJ., Zhang B., An J., Wang JJ., et al. 2010. Genome sequence of the milbemycin-producing bacterium Streptomyces bingchenggensis. Journal of bacteriology, 192(17), pp.4526-4527.

Xu Z., Hao B., 2009. CVTree update: a newly designed phylogenetic study platform using composition vectors and whole genomes. Nucleic acids research, 37(suppl 2), pp.W174-W178. 